\title{
Proton pump inhibitor for eosinophilic duodenal ulcer with esophageal involvement in a 12-year-old boy
}

\author{
YUJI FUJITA $^{1}$, Keiichi Tominaga ${ }^{1}$, Kazuyuki Ishida ${ }^{1}$, Hironori Masuyama ${ }^{2}$, and Shigemi \\ Yoshihara $^{1}$ \\ ${ }^{1}$ Dokkyo Medical University \\ ${ }^{2}$ Masuyama Gastrointestinal Clinic
}

September 25, 2021

\section{Clinical Letters to the Editor}

Proton pump inhibitor for eosinophilic duodenal ulcer with esophageal involvement in a 12year-old boy

Yuji Fujita ${ }^{1}$, MD, Keiichi Tominaga ${ }^{2}, \mathrm{MD}, \mathrm{PhD}$, Kazuyuki Ishida ${ }^{3}, \mathrm{MD}, \mathrm{PhD}$, Hironori Masuyama ${ }^{4}, \mathrm{MD}$, $\mathrm{PhD}$, Shigemi Yoshihara ${ }^{1}, \mathrm{MD}, \mathrm{PhD}$

\section{Affiliations:}

1. Department of Pediatrics, Dokkyo Medical University, Tochigi, Japan

2. Department of Gastroenterology, Dokkyo Medical University, Tochigi, Japan

3. Department of Diagnostic Pathology, Dokkyo Medical University, Tochigi, Japan

4. Masuyama Gastrointestinal Clinic, Tochigi, Japan

\section{Corresponding author:}

Yuji Fujita, MD

Department of Pediatrics, Dokkyo Medical University, 880 Kitakobayashi, Mibu, Shimotsuga, Tochigi 3210293, Japan

Phone: 81-282-86-1111, Fax: 81-282-86-7521

Email: fujitay@dokkyomed.ac.jp

Funding Sources: This research did not receive any specific grant from funding agencies in the public, commercial, or not-for-profit sectors.

Financial Disclosure: The authors declare they have no relevant financial relationships.

Conflict of Interest Statement: The authors declare they have no conflicts of interest.

Author contributions: Y.F. collected and analyzed the data and drafted and revised the initial manuscript. K.T., K.I., H.M., and S.Y. interpreted all the data and critically revised the manuscript for important intellectual content. All the authors approved the final manuscript and agreed to be accountable for all aspects of the work.

To the Editor,

A 13-year-old boy with no relevant family or medical history presented with a one-month history of epigastric pain. Laboratory tests indicated a white blood cell count of $4700 / \mu \mathrm{L}$; eosinophil count, $150 / \mu \mathrm{L}(3.2 \%)$; 
albumin, $4.5 \mathrm{~g} / \mathrm{dL}$; C-reactive protein, $<0.01 \mathrm{mg} / \mathrm{dL}$; and total IgE, $151.6 \mathrm{IU} / \mathrm{L}$. The specific IgE antibody levels to the following antigens were tested: egg white, $0.41 \mathrm{IU} / \mathrm{L} ;$ milk, $0.37 \mathrm{IU} / \mathrm{L}$; wheat, $0.25 \mathrm{IU} / \mathrm{L}$; soy, $<0.10 \mathrm{IU} / \mathrm{L}$; peanut, $0.16 \mathrm{IU} / \mathrm{L}$; and salmon, $<0.10 \mathrm{IU} / \mathrm{L}$. Additionally, thymus and activationregulated chemokine levels were at $522 \mathrm{pg} / \mathrm{mL}$, and the IgG antibody forHelicobacter pylori was absent. Abdominal ultrasonography depicted no abnormal findings such as ascites. Esophagogastroduodenoscopy (EGD) revealed longitudinal linear furrows in the esophagus, indicating eosinophilic esophagitis with an A1 ulcer from the duodenal bulb to the descending duodenum, and no abnormal findings in the stomach (Fig. 1A and B). The rapid urease test result was negative. Colonoscopy and balloon-assisted enteroscopy revealed no abnormalities in the ileum and colon. Pathological examination illustrated eosinophilic infiltration of 60 per high-power field (/HPF) in the esophagus and more than 100/HPF in the duodenum (Fig. 2A and B), and no eosinophilic infiltration in the ileum and colon. The patient was diagnosed with an eosinophilic duodenal ulcer with esophageal involvement. Esomeprazole, a proton pump inhibitor (PPI), was orally administered, after which his symptoms promptly improved. After 2 months, the EGD (Fig. 1C and D) and pathological examination (Fig. 2C and D) results revealed improvement in both the esophagus and duodenum.

Herein, we describe the first pediatric case of eosinophilic duodenal ulcer with esophageal involvement that was effectively treated by PPI monotherapy. Eosinophilic gastrointestinal disorders (EGIDs) comprise eosinophilic esophagitis (EoE) and eosinophilic gastroenteritis (EGE). ${ }^{1,2}$ Another classification stratifies EGIDs into EoE and non-EoE EGIDs, and further classifies non-EoE EGIDs into the following: eosinophilic gastritis (EG), which is localized in the stomach and esophagus; EGE, which occurs in the stomach and small intestine (with or without esophagus involvement); eosinophilic enteritis (EE), which is localized in the small intestine; and eosinophilic colitis (EC), which is localized in the colon. ${ }^{3}$ However, none of the classifications consider cases of eosinophilic inflammation in the gastrointestinal tract below the esophagus as EoE. The rate of EGE with esophageal involvement is reported to be $9 \%$ according to a Japanese study ${ }^{2}$. Yamamoto et al. ${ }^{3}$ also reported that EE and EC had no esophageal lesions, but $49 \%$ of EGE cases had esophageal lesions. To date, there have been no reports of an eosinophilic duodenal ulcer with esophageal involvement but without post-duodenal involvement at the time of diagnosis. The possibility of EGIDs should be investigated in case of duodenal ulcers by an active biopsy, and other gastrointestinal lesions should be probed for. ${ }^{2-4}$

A definitive treatment protocol for eosinophilic duodenal ulcer with esophageal involvement remains unestablished. Although PPIs are recommended for EoE as the first line treatment, ${ }^{5,6}$ the effectiveness of PPI for esophageal involvement in non-EoE EGIDs has not been investigated. However, some case studies have reported the effectiveness of PPI for eosinophilic duodenal ulcers. ${ }^{7}$ Systemic glucocorticoids, which are associated with various side effects, especially in children, are more often indicated in non-EoE EGIDs than in $\mathrm{EoE}^{2,3}$. Elimination dietary treatment may be useful in non-EoE EGIDs; however, it is not an easy treatment as it can lead to failure to thrive in children. In contrast, PPI have fewer side effects and can be used safely. In conclusion, PPI monotherapy may be considered as a first line treatment for eosinophilic duodenal ulcers with esophageal involvement, depending on the severity.

\section{List of abbreviations:}

EG: eosinophilic gastritis

EGD: Esophagogastroduodenoscopy

EoE: eosinophilic esophagitis

EGE: eosinophilic gastroenteritis

EGID: Eosinophilic gastrointestinal disorder

HPF: high-power field

PPI: proton pump inhibitor

Acknowledgments: We would like to thank Editage (www.editage.com) for English language editing. 


\section{References}

1. Kinoshita Y, Oouchi S, Fujisawa T. Eosinophilic gastrointestinal diseases - Pathogenesis, diagnosis, and treatment. Allergol Int 2019; $68: 420-429$.

2. Kinoshita Y, Furuta K, Ishimaura N, Ishihara S, Sato S, Maruyama R, et al. Clinical characteristics of Japanese patients with eosinophilic esophagitis and eosinophilic gastroenteritis. J Gastroenterol2013; $48: 333-9$.

3. Yamamoto M, Nagashima S, Yamada Y, Takatsugu M, Shimoyama Y, Takahashi S, et al. Comparison of nonesophageal eosinophilic gastrointestinal disorders with eosinophilic esophagitis: A nationwide survey. J Allergy Clin Immunol Pract 2021; S2213-2198 : 00714-5.

4. Yamazaki K, Sakashita T, Iwata H, Mizutani T, Matsuura K, Sugiyama T et al. A case of a teenage boy with eosinophilic gastroenteritis with esophageal involvement developing a hemorrhagic duodenal ulcer.Clin J Gastroenterol 2015; 8 : 179-85.

5. Lucendo AJ, Molina-Infante J, Arias A, von Arnim U, Bredenoord AJ, Bussmann C, et al. Guidelines on eosinophilic esophagitis: evidence-based statements and recommendations for diagnosis and management in children and adults. United European Gastroenterol $J$ 2017; 5 : 335-58.

6. Dellon ES, Liacouras CA, Molina-Infante J, Furuta GT, Spergel JM, Zevit N, et al. Updated International Consensus Diagnostic Criteria for Eosinophilic Esophagitis: Proceedings of the AGREE Conference. Gastroenterology 2018; 155 : 1022-33.

7. Tee HP, Swarts D, Tydd T, Leong RWL. Gastrointestinal: Eosinophilic enteritis manifesting as brownpigmented duodenal ulcers. J Gasroenterol Hepatol 2009; 24 : 1892.

\section{Figure legends}

Figure 1. Esophagogastroduodenoscopy images showing longitudinal linear furrows in the esophagus (A) and a stage A1 ulcer from the duodenal bulb to the descending duodenum (B). These findings improved 2 months after treatment with esomeprazole (C, D).

Figure 2. Pathological examination results showing eosinophil infiltration of 60 per high-power field (/HPF) in the esophagus (A) and more than 100/HPF in the duodenum (B). The eosinophil infiltration improved to $3 / \mathrm{HPF}$ in the esophagus (C) and 10/HPF in the duodenum (D) 2 months after initiation of treatment with esomeprazole. (A-D, original magnification, x400)

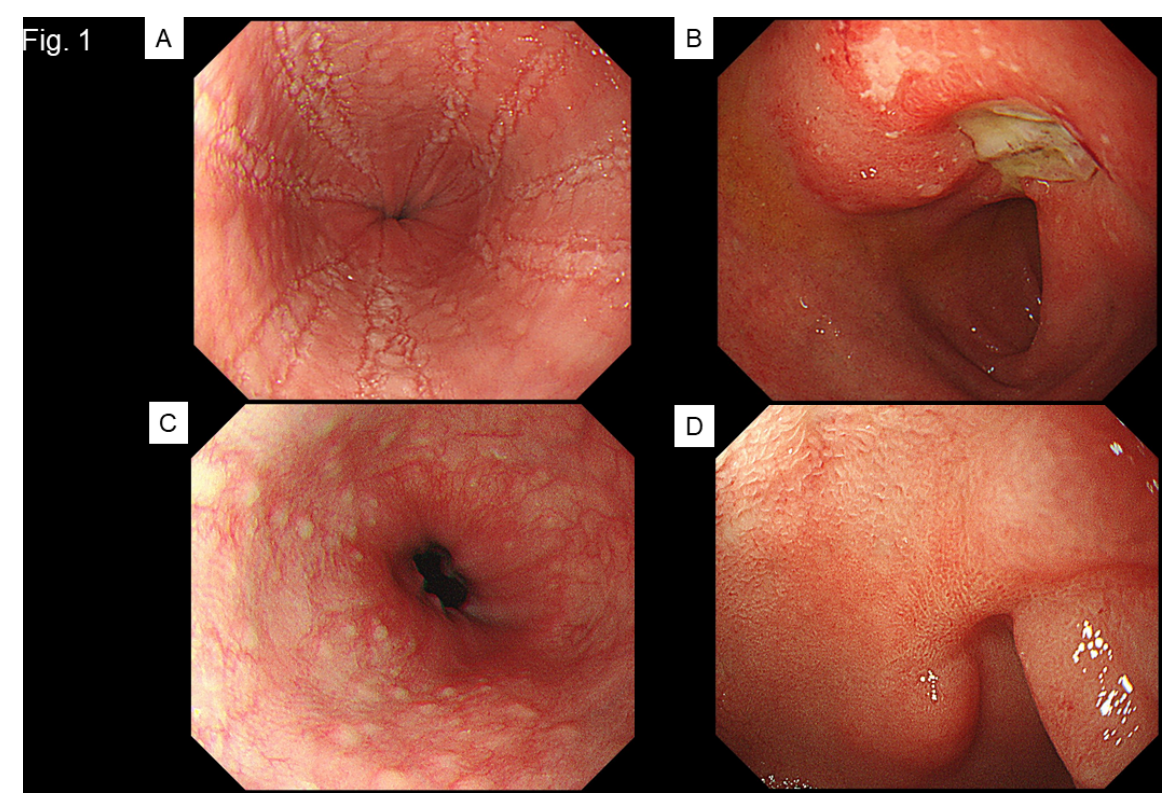




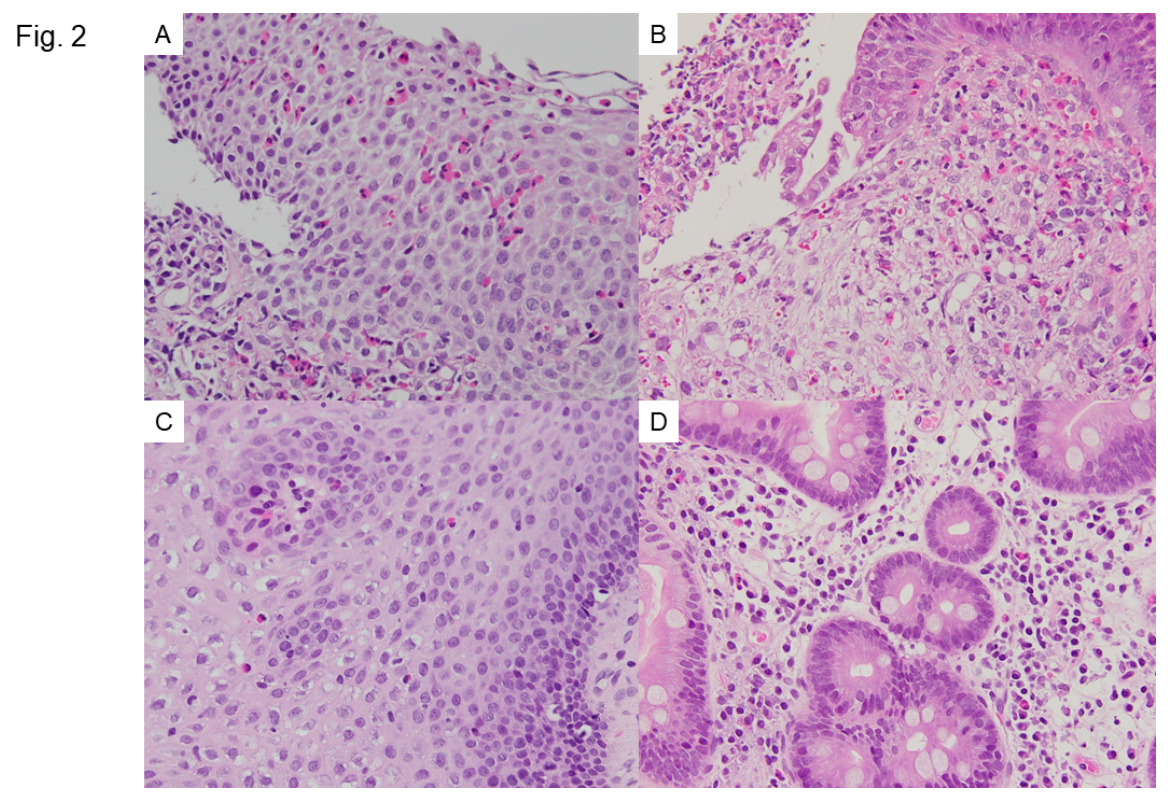

